\title{
THE DESIGN OF EFFICIENT STATED CHOICE EXPERIMENTS
}

\author{
STEPHEN BUSH
}

(Received 8 April 2010)

2000 Mathematics subject classification: primary 62K05; secondary 05B15.

Keywords and phrases: optimal design, orthogonal array, Davidson ties model, Davidson-Beaver position effects model, multinomial logit model.

Making choices is a fundamental part of life. Whether it be the food that we eat, how we get from $\mathrm{A}$ to $\mathrm{B}$, or the things that we do or do not purchase, choices are made all of the time. The ability to understand and influence these choices is valuable in many areas such as marketing, health economics, tourism, transportation research, and public policy. Choice experiments allow researchers in these areas to show respondents sets of options, described by attributes, and use the attributes of the chosen options to determine how important each of the attributes is to the 'attractiveness' of any option. From this information market share or policy acceptability can be predicted.

In this thesis we look at optimal designs for the multinomial logit (MNL) model, and for two extensions of this model. The first extension incorporates tied preferences, and is based on the extension of the Bradley-Terry model introduced by [1]. The second extension allows the researcher to estimate the effect that the position of an item in the set of alternatives has on the perceived merit of the item. This extension is based on the extension of the Bradley-Terry model introduced by [2]. We prove results that give optimal designs, both for the extensions of the Bradley-Terry model and the extensions of the MNL model, and conduct simulations of these models. Finally, we prove results that give optimal designs for the MNL model when the starting design is an orthogonal array constructed using the Rao-Hamming construction, rather than a complete factorial design.

Thesis submitted to the University of Technology, Sydney, June 2009. Degree approved, February 2010. Supervisor: Professor Deborah Street.

(C) 2010 Australian Mathematical Publishing Association Inc. 0004-9727/2010 \$16.00 


\section{References}

[1] R. R. Davidson, 'On extending the Bradley-Terry model to accommodate ties in paired comparison experiments', J. Amer. Stat. Assoc. 65 (1970), 317-328.

[2] R. R. Davidson and R. J. Beaver, 'On extending the Bradley-Terry model to incorporate withinpair order effects', Biometrics 33 (1977), 693-702.

STEPHEN BUSH, Department of Mathematical Sciences, University of Technology, Sydney, PO Box 123 Broadway NSW 2007, Australia

e-mail: stephen.bush@uts.edu.au 